\title{
Stabilization of Polymer Nanocomposites in High-Temperature and High-Salinity Brines
}

\author{
Maje Alhaji Haruna ${ }^{\dagger}$ a and Dongsheng Wen*, ${ }^{\dagger},+$ (]) \\ ${ }^{\dagger}$ School of Chemical and Process Engineering, University of Leeds, Leeds LS2 9JT, U.K. \\ ${ }^{\ddagger}$ School of Aeronautic Science and Engineering, Beihang University, Beijing 100083, P. R. China
}

Supporting Information

ABSTRACT: Stabilization of polymer nanocomposites in aqueous environment with high salinity has been a constant challenge for their applications. This work aimed to improve the stability of graphene oxide (GO) polyacrylamide nanocomposites at high-temperature and high-ionic-strength brines. GO was synthesized via a modified Hummers' method and the copolymer of acrylamide (COPAM) was obtained via free-radical polymerization. The covalent functionalization of COPAM with the partially reduced GO (rGO) was successfully achieved. 1,3-Propane sultone was used to further functionalize the obtained rGOCOPAM composites to accomplish the zwitterionic character on the rGO-COPAM surface to get a material with excellent temperature stability and dispersibility in the presence of high ionic strength brines. The synthesized materials were characterized by

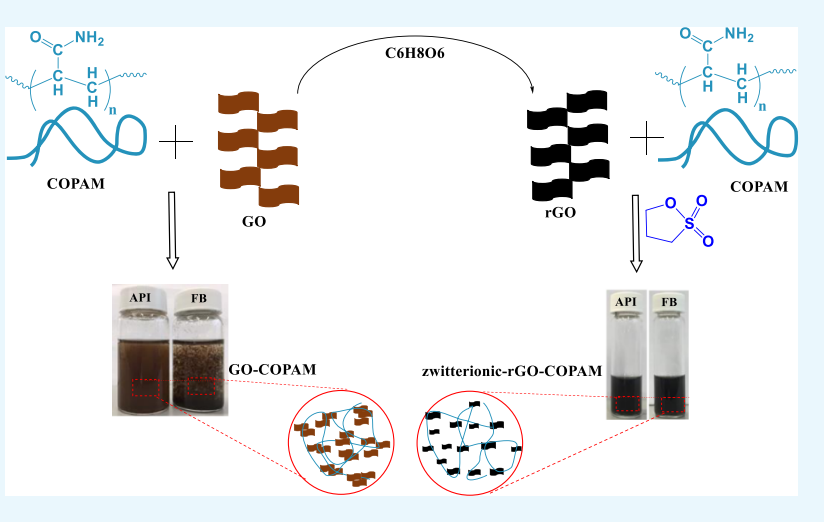
${ }^{1} \mathrm{H}$ NMR, gel permeation chromatography, X-ray photoelectron spectroscopy, scanning electron microscopy, transmission electron microscopy analysis, and so forth. The thermal stability of the dispersion at $80{ }^{\circ} \mathrm{C}$ for 120 days was observed by visual inspection and spectroscopic analysis. The results showed that the zwitterionic polymer produced excellent brine stability with GO nanosheets and suggested promising applications of zwitterionic polyacrylamide-GO systems especially for enhanced oil recovery.

\section{INTRODUCTION}

Polymer nanocomposite stabilization in aqueous environment with extreme salinity (high ionic strength) has been considered recently as a key subject in the research field. For instance, in petroleum industries, nanomaterials have been recently proposed to deliver into oil reservoirs acting as sensors or imaging enhancers to provide useful information regarding the reservoir conditions. However, delivering nanomaterials into a reservoir is a challenging task because of the high salinity and high temperature of the environment, which can easily destabilize the injected nanomaterials.

Several approaches such as physical dispersion methods, covalent bonding, and noncovalent bonding methods have been implemented to address this stabilization issue. A composite of carbon nanoparticles and ionic polymer (poly(vinyl alcohol)) was shown to be stable in American Petroleum Institute brine (APIB). The polymer was grafted onto the nanoparticle surface by acidic treatment using chlorosulfonic acid. It has been reported that the nanoparticles that are partially sulfonated showed better dispersion stability at $100{ }^{\circ} \mathrm{C}$ in APIB than highly sulfonated nanoparticles, while unsulfonated nanoparticles were not dispersed. The long-term temperature stability, however, was not examined, which prevented their use as reservoir sensors. ${ }^{1}$ Vancso et al. also observed the stability of polyimidazole betaine in $22.6 \mathrm{wt} \% \mathrm{NaCl}$ solution without reporting the long-term thermal stability. ${ }^{2}$ Johnston and coworkers ${ }^{3}$ attempted to stabilize nanocomposites containing poly-acrylate-/acrylamide-type polymers and iron oxide nanoparticles wrapped with sulfonic salt in reservoir conditions with APIB for the duration of 1 month at $90{ }^{\circ} \mathrm{C}$. The finding showed that the stabilization was favored by the ionic nature of the polymer and nanoparticle repulsion against agglomeration.

The copolymer of acrylamide (COPAM) is a popular polymer that has extensive use in different areas including oil and gas because of its excellent properties. ${ }^{4-11}$ Several investigations have been conducted on the graphene oxide (GO)based polyacrylamide composites; ${ }^{12-20}$ for example, in oil and gas exploitation and production, it was primarily used as an additive in an advanced drilling fluid for fluid-loss control. ${ }^{21}$ It has been reported that GO improved the rheology and thermal stability of polyacrylamide under a high temperature of up to $85^{\circ} \mathrm{C}^{22}$ this improvement is attributed to the presence of the large number of the anionic group on the edge of the GO sheet, which contributes to the electrostatic repulsion. ${ }^{21-24}$ However, despite the tremendous use of polyacrylamide/GO nanocomposites, its stability in aqueous solution with high

Received: April 4, 2019

Accepted: May 24, 2019

Published: July 5, 2019 
ionic strength is a challenging issue because of GO sheet aggregation and restacking, which is due to the strong intersheet van der Waals forces. ${ }^{25,26}$ However, for EOR applications, these nanocomposites are required to be stable under hightemperature and high-salinity conditions. Our recent preliminary study showed an unsuccessful dispersion stability in GOCOPAM solution at ambient temperature and higher under the influence of both APIB and formation brine (FB). This is probably due to the electrostatic cross-linking between the GO sheets caused by divalent ions and consequently led to destabilization of the solution.

Aiming to address the stability challenges of GO/polyacrylamide nanocomposites in harsh conditions, this work proposed a novel stabilization approach via surface modification of the COPAM and reduced GO (rGO) using 1,3propane sultone in order to accomplish a zwitterionic character on the material surface. It has been reported that zwitterion molecules possess outstanding solubility and stability in the presence of brines because of their antipolyelectrolyte effect. $^{27,28}$ Addition of electrolyte materials results in chain expansion and later promotes stabilization and de-agglomeration via electrostatic chemistry (steric effects). ${ }^{29}$ Zwitterion molecules have been applied as antifouling coatings in seawater ${ }^{30}$ and suggested to be used potentially in enhanced oil recovery. ${ }^{31}$ Recently, the zwitterionic $\mathrm{rGO} /$ poly(vinylimidazole)-co-poly(aminostyrene) composite was reported to be stable at high temperature under the influence of high-ionic-strength brines for over 90 days. $^{28}$

Therefore, a successful GO-COPAM composite was synthesized by covalently attaching the COPAM of acrylamide (AA) and 2-acrylamido-(3-acrylamdopropyl) trimethylammonium chloride (ATAC) monomers onto the partial rGO sheets. The resulting rGO-COPAM composite was further exposed to postfunctionalization chemistry using 1,3-propane sultone to create zwitterion groups (species with functional groups, of which at least one has a positive and one has a negative electrical charge). This approach has an advantage including chemical reactivity between the polymer and large surface area of the GO basal plane, ${ }^{32,33}$ which further promote the functionalization and ability of the adsorbed nanomaterials to be used in the area of interest. $^{34-37}$ The synthesized zwitterionic rGO-COPAM composites were dispersed in APIB and FB, their long-term temperature stability was monitored both spectroscopically and visually upon standing for 120 days at $80{ }^{\circ} \mathrm{C}$. The dispersed zwitterionic rGO-COPAM solution demonstrated excellent dispersion ability in both APIB and FB.

\section{EXPERIMENTAL SECTION}

2.1. Materials. Graphite powder, acrylamide (AA, 99\%), ATAC monomers, 4-4'-azo-bis-4-cyanopentanoic acid (ACPA), hydrogen peroxide $\left(\mathrm{H}_{2} \mathrm{O}_{2}, 36 \%\right)$, potassium permanganate $\left(\mathrm{KMnO}_{4}, 99 \%\right)$, 1,3-propane sultone, sodium nitrite, ascorbic acid, and all salt components were ordered from Sigma-Aldrich, and sulfuric acid $\left(\mathrm{H}_{2} \mathrm{SO}_{4}, 95 \%\right)$ and nitric acid $\left(\mathrm{HNO}_{3}, 69 \%\right)$ were obtained from VWR chemicals. No further purification was performed on the chemicals before use.

2.2. Synthesis of GO. Modified Hummers' method was used to synthesize stable GO nanosheets. ${ }^{38}$ Approximately $20 \mu \mathrm{m}$ of graphite powder was used as the starting material. Nitric and sulfuric acids were mixed in water and used for oxidation of graphite solution. Graphite powder (12 g) was dissolved in a mixture of $50 \mathrm{~mL}$ of $\mathrm{HNO}_{3}$ and $100 \mathrm{~mL}$ of $\mathrm{H}_{2} \mathrm{SO}_{4}$ and stirred at ambient condition for $24 \mathrm{~h}$. Subsequently, distilled water $(100 \mathrm{~mL})$ was added to the mixture followed by continuous stirring for $24 \mathrm{~h} . \mathrm{KMnO}_{4}(12 \mathrm{~g})$ was gently included in the solution and then stirred for another $5 \mathrm{~h}$ under ambient temperature. To keep the temperature low, an ice back containing $60 \mathrm{~mL}$ of $36 \% \mathrm{H}_{2} \mathrm{O}_{2}$ solution was added and stirred overnight. A Whatman filter paper was used to extract the resulting suspension. The filtrate was washed three times using $10 \%$ HCL after centrifugation for $1 \mathrm{~h}$ at $13000 \mathrm{rpm}$, and the supernatant was discarded.

2.3. Synthesis of COPAM. The COPAM and ATAC were synthesized via free-radical polymerization. ${ }^{39,40}$ The polymerization was carried out in a three-neck bottom reactor, equipped with a reflux condenser, nitrogen inlet gas, and a mechanical stirrer as shown in Figure S1. Initially, the reactor was charged with a dissolved mixture of AA and ATAC monomers in $80 \mathrm{~mL}$ of degassed and distilled water. An oil bath was prepared, and the reactor was placed inside. The solution was sprinkled under inert atmosphere for $30 \mathrm{~min}$ while stirring. Water-soluble ACPA initiator (10 mg) was added to the reactor to trigger the reaction. The mixture undergoes $6 \mathrm{~h}$ of heating at $80{ }^{\circ} \mathrm{C}$ while string and then cooled down at room temperature to obtain the clear and viscous product. The solution was precipitated into acetone and allowed to dry overnight under vacuo at $60{ }^{\circ} \mathrm{C}$.

2.4. Synthesis (Preparation) of GO Polymer Composites. GO was dispersed in distilled water to obtain $1 \mathrm{mg} / \mathrm{mL}$ with up to $30 \mathrm{~mL}$ of total solution. In order to obtain partial rGO, $15.1 \mathrm{mg}$ of L-ascorbic acid was included in the solution, followed by $1 \mathrm{~h}$ gentle stirring at $60{ }^{\circ} \mathrm{C}$, and the solution was cooled down and used immediately in the next stage. In a separate container, $3 \mathrm{mg} / \mathrm{mL}$ of COPAM was dissolved in $50 \mathrm{~mL}$ of water while stirring for $5 \mathrm{~min}$ under sonication and allowed to cool down. The cooled rGO dispersion was then slowly added to the COPAM solution, followed by $30 \mathrm{~min}$ of stirring. The mixture of rGO-COPAM was sonicated for $30 \mathrm{~min}$, followed by overnight stirring at $60{ }^{\circ} \mathrm{C}$, and later reduced in vacuo (concentrated). Subsequently, the solution was centrifuged for $15 \mathrm{~min}$ at $5000 \mathrm{rpm}$ to induce flocculation after acetone was added. The product was freeze-dried to obtain a solid rGO-COPAM composite.

2.5. Synthesis of Zwitterionic Polymer Composites. The zwitterionic rGO-COPAM composites were prepared by modification using 1,3 propane sultone following the procedure published in the previous literature. ${ }^{28}$ The amount of 1,3 propane sultone was experimentally decided after conducting many experiments to determine the effect of different 1,3 propane sultone concentrations in COPAM solubility and viscosity. Different concentrations of 1,3 propane sultone $(0.05,0.15,0.25,0.35,0.45$, and 0.55 wt \%) were dispersed into the COPAM solution followed by gentle stirring at room temperature and observed their changes in solubility overnight; their viscosity was also subsequently measured. However, both the solubility (Figure S2a) and viscosity (Figure $\mathrm{S} 2 \mathrm{~b}$ ) remain the same in all the concentrations. Therefore, the amount of 1,3 propane sultone was selected based on the previously published procedure. ${ }^{28}$ rGO-COPAM was dispersed in $100 \mathrm{~mL}$ of water under sonication at room temperature, followed by the addition of $350.2 \mathrm{mg}$ of 1,3-propane sultone. ${ }^{28}$ The above combination was then transferred into a three-neck bottom flask and refluxed overnight under inert atmosphere. The mixture undergoes $15 \mathrm{~min}$ centrifugation at a speed of $2500 \mathrm{rpm}$ and then the supernatant was decanted. The resulting solution was 
Table 1. Variation in the Salt Components of Brine Mixtures

$\begin{array}{llc}\text { salt components } & \text { FB (\%) } & \text { APIB (\%) } \\ \mathrm{MgCl}_{2} \cdot 6 \mathrm{H}_{2} \mathrm{O} & 1.3 & \\ \mathrm{NaCl} & 7.5 & 8 \\ \mathrm{Na}_{2} \cdot \mathrm{SO}_{4} & 0.006 & \\ \mathrm{NaHCO}_{4} & 0.005 & \\ \mathrm{BaCl}_{2} & 0.001 & \\ \mathrm{CaCl}_{2} \cdot 2 \mathrm{H}_{2} \mathrm{O} & 5.0 & 2\end{array}$

then cleaned with methanol and distilled water (1:9). The obtained zwitterionic rGO-COPAM composite was sealed and stored in a clean container after removing methanol in vacuo.

2.6. Preparation of Brine Solutions. APIB was obtained by dissolving $5.0 \mathrm{~g}$ of calcium chloride dihydrate and $20.0 \mathrm{~g}$ of sodium chloride in $225 \mathrm{~mL}$ of distilled water. FB was achieved by dissolving $24.9 \mathrm{~g}$ of calcium chloride dehydrate, $37.3 \mathrm{~g}$ of sodium chloride, $0.26 \mathrm{~g}$ of sodium bicarbonate, $0.3 \mathrm{~g}$ of sodium sulfate, $6.6 \mathrm{~g}$ of magnesium chloride hexahydrate, and $5 \mathrm{mg}$ of barium chloride in $500 \mathrm{~mL}$ of distilled water. The percentages of individual salts are shown in Table 1.

2.7. Stability of Zwitterionic rGO-COPAM in Brine Solutions. First, $90 \mathrm{~mL}$ of the respective brine solutions was added into each of the two separate capped containers labeled APIB and FB containing $10 \mathrm{~mL}$ of zwitterionic rGO-COPAM composite to obtain a 9:1 ratio. After sonicating the solutions for $5 \mathrm{~min}$, it was then stored at $80{ }^{\circ} \mathrm{C}$ uninterrupted in an oven. A portion of each sample was taken for characterization prior to the storage and after $24 \mathrm{~h}$ ( 1 day) and then subsequently after the intervals of $10,30,60,90$, and 120 days. The photos of the extracted solutions were taken on those days for comparison as shown in Figure 6.

2.8. Characterization. The proton nuclear magnetic resonance (NMR) of the polymer was carried out on a JEOL-600 NMR spectrometer. Deuterium oxide $\left(\mathrm{D}_{2} \mathrm{O}\right)$ solvent was used for field-frequency lock, and the observed proton chemical shifts are reported in parts per million. The Agilent Technologies Infinity gel permeation chromatography (1260 MDS, GPC) was applied for determining the molecular weight of the polymer. $\mathrm{NaNO}_{3}(0.1 \mathrm{M})$ was used as the mobile phase for the molecular weight and polydispersity analysis. The samples were filtered by $0.22 \mu \mathrm{m}$ pore size GVWP hydrophilic membrane before injection. The functionality of the samples was measured by attenuated total reflection (ATR)-Fourier transform infrared (FTIR, Nicolet iS10). The data were recorded within the frequency range of $4000-400 \mathrm{~cm}^{-1}$ at a spectral resolution of $4 \mathrm{~cm}^{-1}$. The percentage of chemical composition was determined on an elemental (CHNS-O) analyzer (CHNS-O analyzer, Thermo Scientific FLASH 2000). X-ray photoelectron spectroscopy (XPS) was also used to evaluate the elemental analysis for prepared composites. The thermal stability was performed using a DSC-3/ TGA/(Mettler Toledo) star ${ }^{\mathrm{e}}$ system. It was operated under nitrogen gas at $50 \mathrm{~mL} / \mathrm{min}$ flow rate and heating range

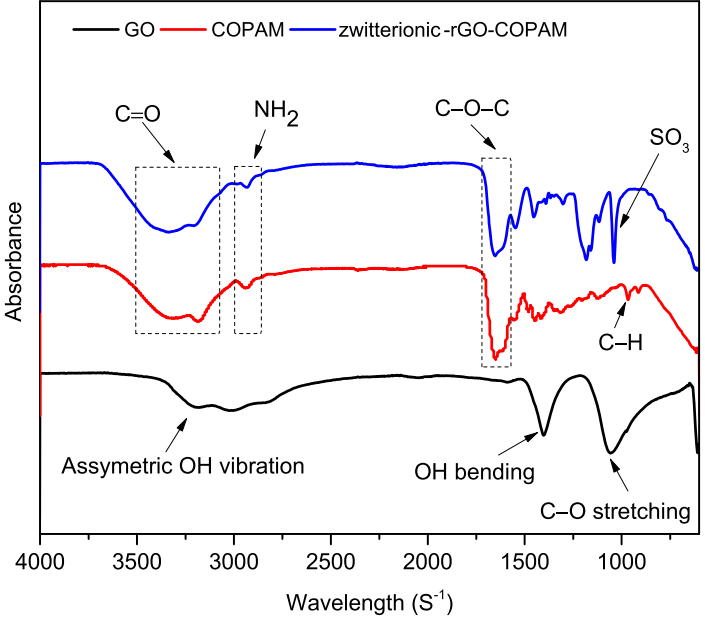

Figure 1. FTIR spectra of GO, COPAM, and zwitterionic rGOCOPAM nanocomposites.

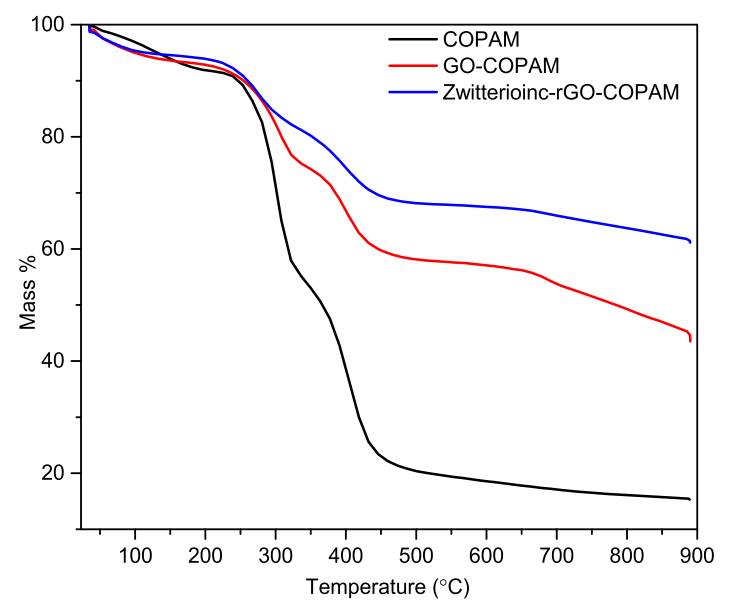

Figure 2. TGA profiles of COPAM, GO-COPAM and zwitterionicrGO-COPAM nanocomposites.

between 35 and $900{ }^{\circ} \mathrm{C}$ with an interval of $10{ }^{\circ} \mathrm{C} / \mathrm{min}$. A scanning electron microscope with high-performance coldfield emission (SU8230 Hitachi, Leeds Electron Microscopy and Spectroscopy Centre, UK) operated at $2 \mathrm{kV}$ and a transmission electron microscope operated at 300 (FEI Titan Themis Cubed 300, Leeds Electron Microscopy and Spectroscopy Centre, UK) were used to observe the surface morphology. An energy-dispersive X-ray (EDX) spectroscopy (Oxford INCA 350) attached to the scanning electron microscope was used to identify the elemental compositions and location of the element. The sedimentation behavior of both GO-COPAM and zwitterionic rGO-COPAM composites was evaluated after the initial preparation stage in APIB and FB using Turbiscan, a vertical scan analyzer (MA 2000, Toulouse, France), using the procedure reported in the literature. ${ }^{41}$ The percent transmittance ( $\% T$ ) spectra of zwitterionic $\mathrm{rGO}-\mathrm{COPAM}$ solution in both APIB and FB

Table 2. Properties and Elemental Compositions of the Polymer

\begin{tabular}{|c|c|c|c|c|c|c|c|c|}
\hline \multirow[b]{2}{*}{ properties } & \multirow[b]{2}{*}{$M_{\mathrm{w}}(\mathrm{g} / \mathrm{mol})$} & \multirow[b]{2}{*}{$M_{\mathrm{n}}(\mathrm{g} / \mathrm{mol})$} & \multirow[b]{2}{*}{ PDI } & \multicolumn{5}{|c|}{ elemental compositions (\%) } \\
\hline & & & & $\mathrm{C}$ & $\mathrm{N}$ & $\mathrm{H}$ & $\mathrm{O}$ & $S$ \\
\hline COPAM & 343140 & 297457 & 2.14 & 47.96 & 15.63 & 8.66 & 26.73 & 0 \\
\hline zwitterionic rGO-COPAM & & & & 37.36 & 10.77 & 5.88 & 44.56 & 1.43 \\
\hline
\end{tabular}



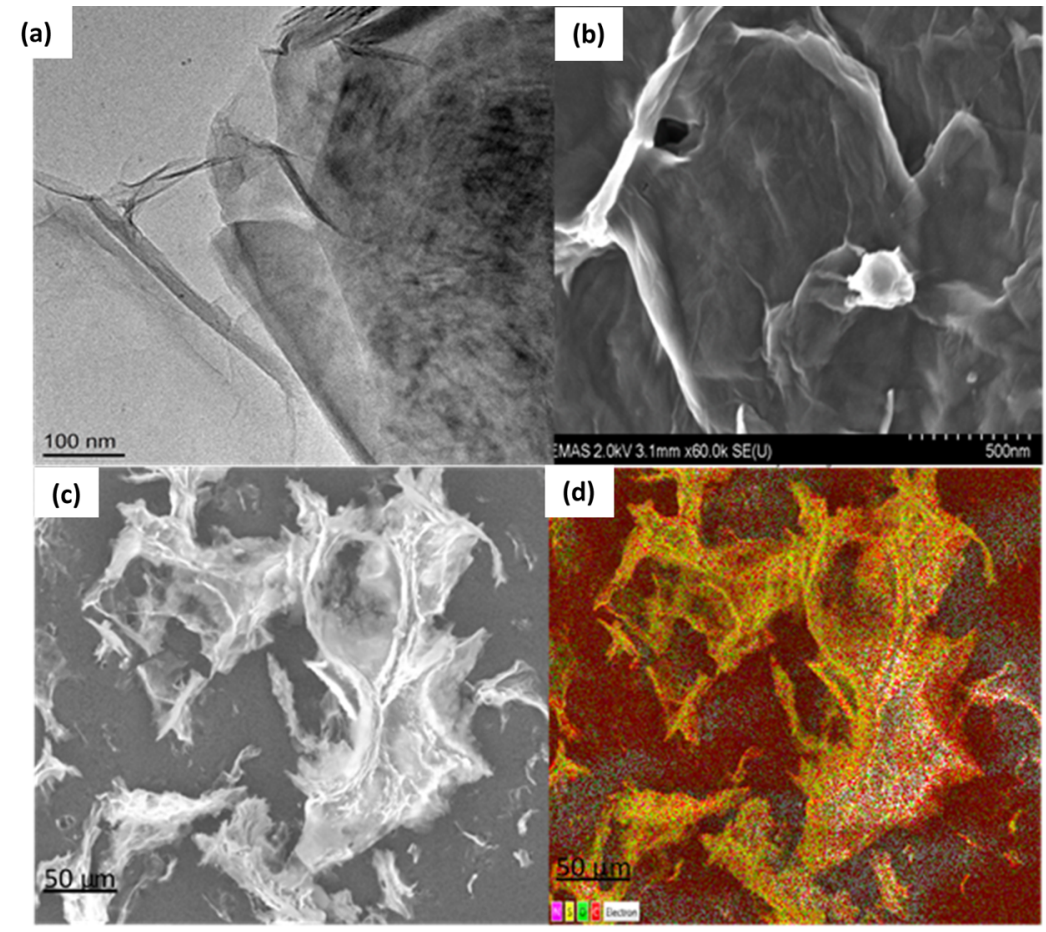

(e)
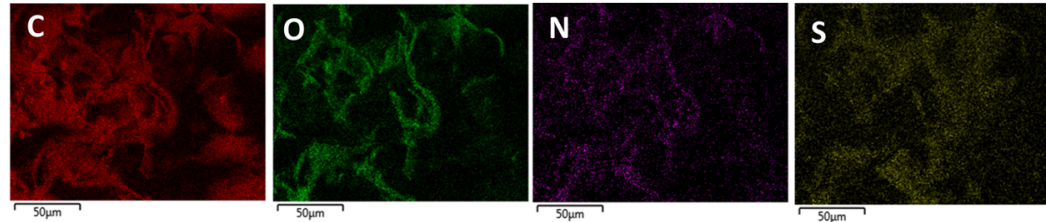

Figure 3. (a) TEM and (b) SEM images of GO nanosheets synthesized via modified Hummer's method, (c) SEM image of zwitterionic rGOCOPAM nanocomposites, (d) SEM mapping of zwitterionic rGO-polyacrylamide sample, where the elements are represented by different colors (pink; nitrogen, yellow; sulfur, green; oxygen; and red; carbon), and (e) elemental analysis of zwitterionic rGO-COPAM extracted from SEM mapping.

samples were recorded using Varian Cary 6000i UV-vis spectroscopy in quartz cuvettes. The zeta potential together with the particle size distribution was measured by dynamic light scattering (DLS) technique using a Malvern Nanosizer. The dispersion stability of zwitterionic rGO-COPAM composite was observed to investigate the sedimentation and flocculation behavior by measuring the transmission of nearinfrared light under the influence of centrifugal force using (LUMiSizer, Lum GmbH, Germany) a dispersion analyzer centrifuge. The measurement was conducted by pipetting $400 \mu \mathrm{L}$ of the sample into a polycarbonate tube cell. The tube was then inserted into an equipment to measure the sedimentation profiles along the sample length during the centrifugation. The software attached to the system recorded the real-time transmission profiles at the certain intervals.

\section{RESULTS AND DISCUSSION}

3.1. Characterizations. The polydispersity index (PDI), weight, and number-average molecular weight $\left(M_{\mathrm{w}}\right.$ and $\left.M_{\mathrm{n}}\right)$ of the polymer observed using GPC are shown in Table 2. The ${ }^{1} \mathrm{H}$ NMR spectra of the COPAM were recorded after the sample was dissolved in $\mathrm{D}_{2} \mathrm{O}$, as shown in Figure $\mathrm{S} 3$. The peak at $1.4 \mathrm{ppm}$ and around 3.1-3.7 ppm showed the presence of methyl and ethylene protons in the polymer units. The peaks between 2.0 and $2.4 \mathrm{ppm}$ indicate the existence of methane $\left(-\mathrm{CH}_{3}\right)$ in the polymer backbone. $\mathrm{R}-\mathrm{CO}-\mathrm{NH}_{2}$ and $\mathrm{C}-\mathrm{H}$ absorption peaks were observed at 7.6 and $6.9 \mathrm{ppm}$, revealing the formation of major polyacrylamide functional groups in the polymer spectrum. ${ }^{42}$ The XPS (Figure S4) and CHNS-O (Table 2) analysis of the dried samples confirmed that $\mathrm{C}, \mathrm{N}$, and $\mathrm{O}$ are present in both GO-COPAM and zwitterionic $\mathrm{rGO}-$ COPAM composites, with the addition of sulfur in the zwitterionic rGO-COPAM sample, which confirmed the successful functionalization of the material using 1,3-propane sultone.

Figure 1 depicted the ATR-FTIR spectra of GO, COPAM, and zwitterionic rGO-COPAM composites. The spectrum of GO sheets displayed the bands at 1069.7, 1395.5, and $3420 \mathrm{~cm}^{-1}$, representing $\mathrm{C}-\mathrm{O}$ stretching, $\mathrm{O}-\mathrm{H}$ bending, and $\mathrm{O}-\mathrm{H}$ asymmetric/symmetric vibrations of the GO epoxide group. ${ }^{43,44}$ In the COPAM spectrum, it has been observed that the vibration peak at $967 \mathrm{~cm}^{-1}$ is representing the quaternary ethoxylated group of ammonium molecules in the ATAC cationic monomer. ${ }^{45,46}$ The peaks at 1649 and $1117 \mathrm{~cm}^{-1}$ denoted the adsorption bands of $\mathrm{C}-\mathrm{O}-\mathrm{C}$ bonds and $\mathrm{C}-\mathrm{H}$ stretching vibrations, the 3321 and $1700 \mathrm{~cm}^{-1}$ peaks correspond to the carbonyl $(\mathrm{C}=\mathrm{O})$ functionalities, while the amide vibrational bands emerged around 2923 and $1548 \mathrm{~cm}^{-1}$, respectively. ${ }^{47}$ Contrarily, the spectrum of the zwitterionic rGO-COPAM sample showed the combination of peaks found in GO and COPAM samples. However, in the GO spectrum, the peak at $1069.7 \mathrm{~cm}^{-1}$ became weaker and the peak at $1395.5 \mathrm{~cm}^{-1}$ shifted to the higher wavenumber, which might 
At preparation stage After 1 day aging at $80^{\circ} \mathrm{C}$

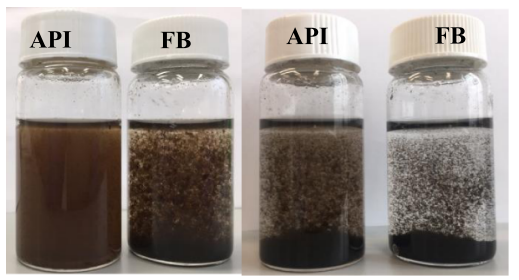

Figure 4. GO-COPAM dispersion in APIB and FB showing an unstable mixture after preparation stage and 1 day of aging at $80^{\circ} \mathrm{C}$.

be due to the hydrolyzable covalent cross-links formed between COPAM and GO. ${ }^{48,49}$ Similarly, the peaks observed at 2923 and $3321 \mathrm{~cm}^{-1}$ in the COPAM spectra were also observed in the composites while shifted slightly to lower wavelengths (2859 and $3302 \mathrm{~cm}^{-1}$ ), elucidating the formation of hydrogen bonds. The additional adsorption peak at $1040 \mathrm{~cm}^{-1}$ on the composite materials corresponds to the $\mathrm{SO}_{3}$ group from 1,3-propane sultone, leading to the formation of anionic character of the zwitterionic $\mathrm{rGO}-\mathrm{COPAM}$ structure that promotes the dispersion stability in the presence of high ionic strength brines. ${ }^{28,50,51}$ TGA was carried out between 25 and $900{ }^{\circ} \mathrm{C}$ heating, the TGA curves of COPAM, GO-COPAM, and zwitterionic rGO-COPAM samples are shown in Figure 2. The TGA analysis demonstrated that between 50 and $150{ }^{\circ} \mathrm{C}$, all the three samples displayed a negligible weight loss, as a result of the surface desorption of water molecules. It can also be observed that compared to COPAM and GO-COPAM samples, the zwitterionic rGO-COPAM composites show smaller weight loss overall, indicating a highly stable material at harsh conditions, which is due to the presence of the $\mathrm{SO}_{3}$ group of zwitterion character. However, the weight lost on COPAM is roughly divided into three stages: at around $400{ }^{\circ} \mathrm{C}$, almost $20 \%$ of the sample weight was lost; at around $700{ }^{\circ} \mathrm{C}$, almost $12 \%$ loss was observed; and more than $3 \%$ weight loss was observed after heating up to $900{ }^{\circ} \mathrm{C}$, corresponding to the minor disintegration of the surface components on the polymer. Contrarily, the neat polymer showed significant loss of weight $(\sim 45 \%)$ until $350{ }^{\circ} \mathrm{C}$, probably because of the polymer decompositions at elevated temperature, and more weight loss followed on the same material to around $80 \%$ from $450{ }^{\circ} \mathrm{C}$ and above, which is because of ammonia evolution and chain scission of the polymer at high temperature. ${ }^{52-54}$ Overall, the zwitterionic rGO-COPAM composites have high-temperature durability because of the covalent functionalization that occurred between the COPAM and rGO.

The TEM and SEM images of the GO nanosheets are shown in Figure 3a,b, the TEM images show exemplarily crumble and wrinkle-like nature of GO. The SEM image described the smooth surface of GO, confirming that the sheets in GO are linked to one another. Figure $3 \mathrm{c}, \mathrm{d}$ shows the SEM structure of the freeze-dried zwitterionic rGO-COPAM composites showing the stable dispersion and microstructure of materials, indicating that the polymer has been grafted to the GO sheets, confirming the appearance of chemical bonds in the composites. Moreover, the superimposition of GO with the COPAM confirms that the zwitterionic rGO-COPAM interaction is not only a physical contact but also a strong chemical adhesion. ${ }^{28,55}$ SEM-EDX reaffirm the existence of $\mathrm{C}, \mathrm{N}, \mathrm{O}$, and $\mathrm{S}$ in the composite material, as illustrated in Figure $3 \mathrm{e}$. The presence of $\mathrm{C}, \mathrm{O}, \mathrm{N}$, and $\mathrm{S}$ is also confirmed by elemental analysis, and the percentage of each element is shown in Table 2. Interestingly, the presence of $S$ in the EDS mapping and elemental analysis

Scheme 1. Schematic of Synthesis Methods of COPAM, GO-COPAM, and Zwitterionic rGO-COPAM Composites

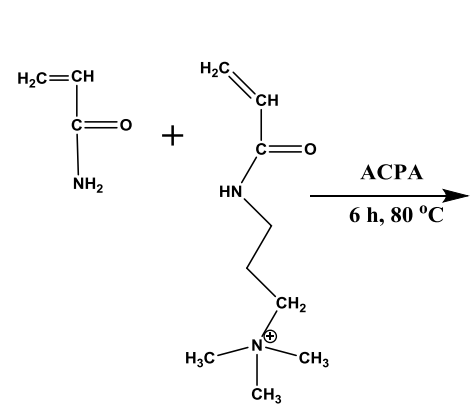

Stage 1

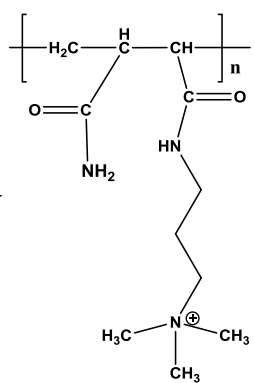

$\mathrm{CH}_{3}$

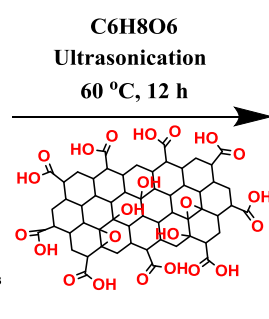

Stage 2
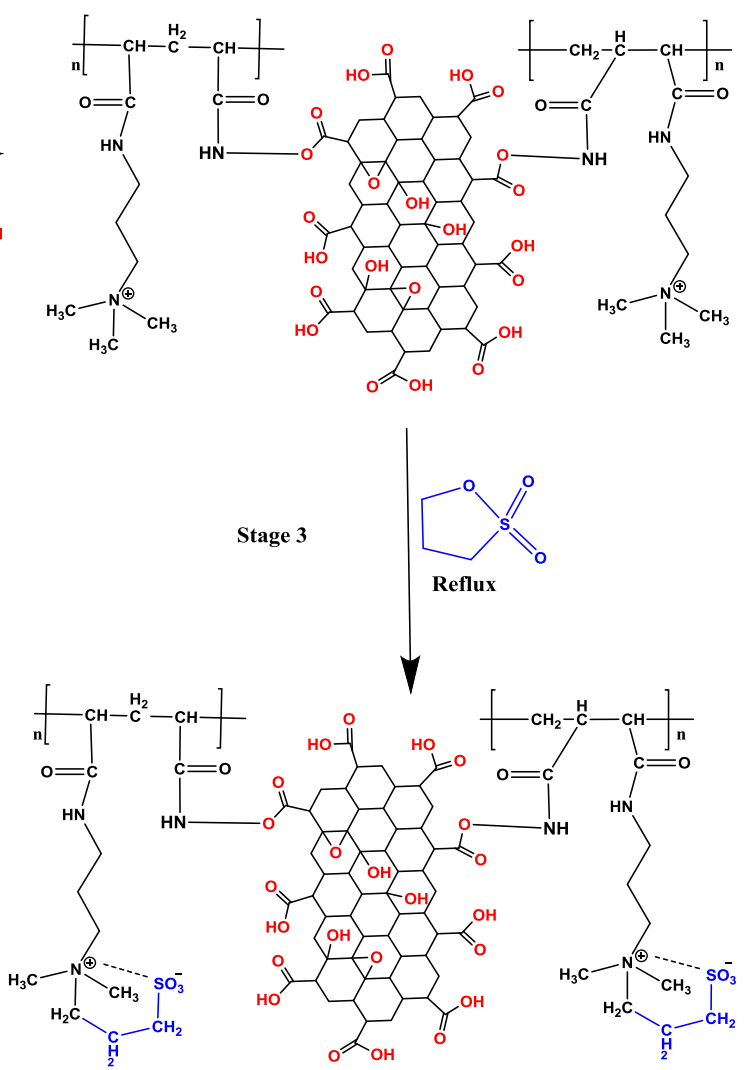
(a)
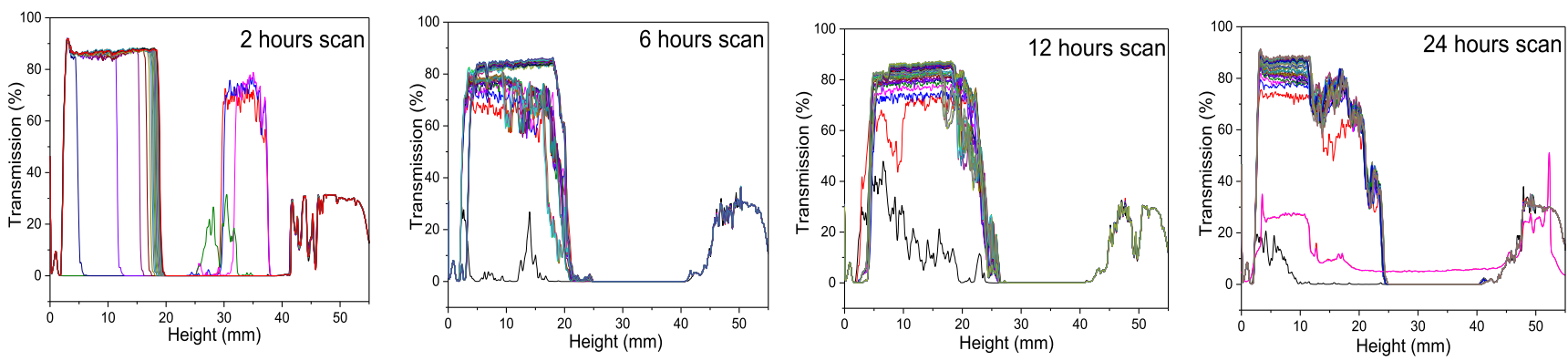

(b)
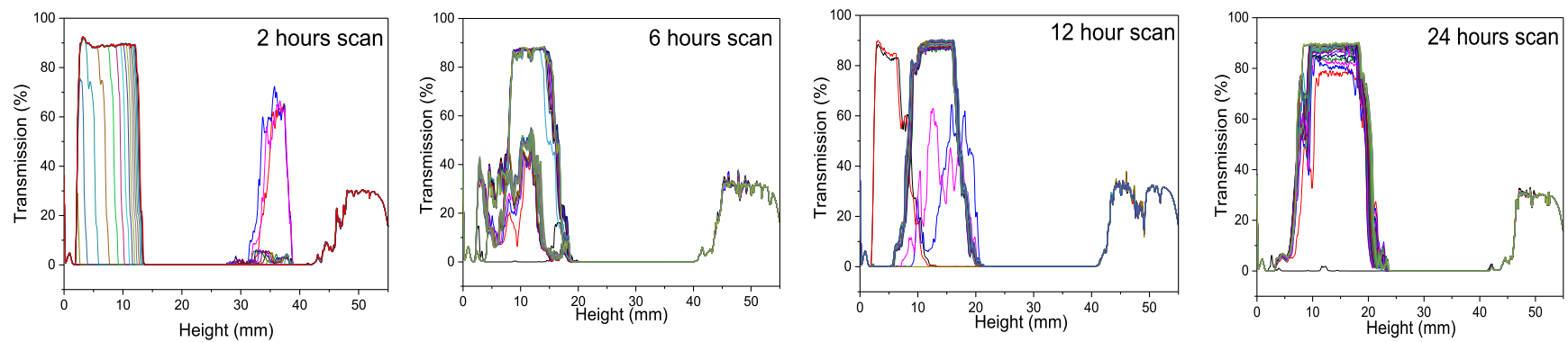

(c)
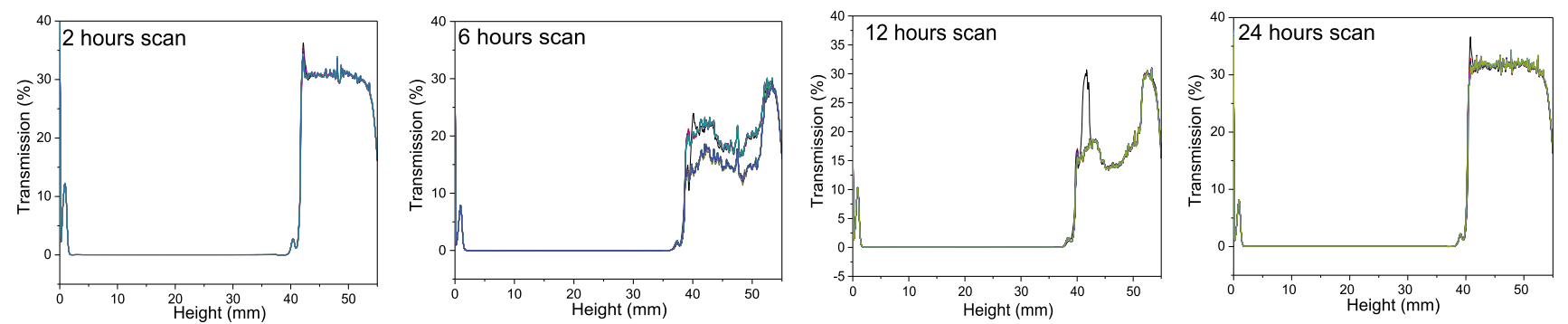

(d)
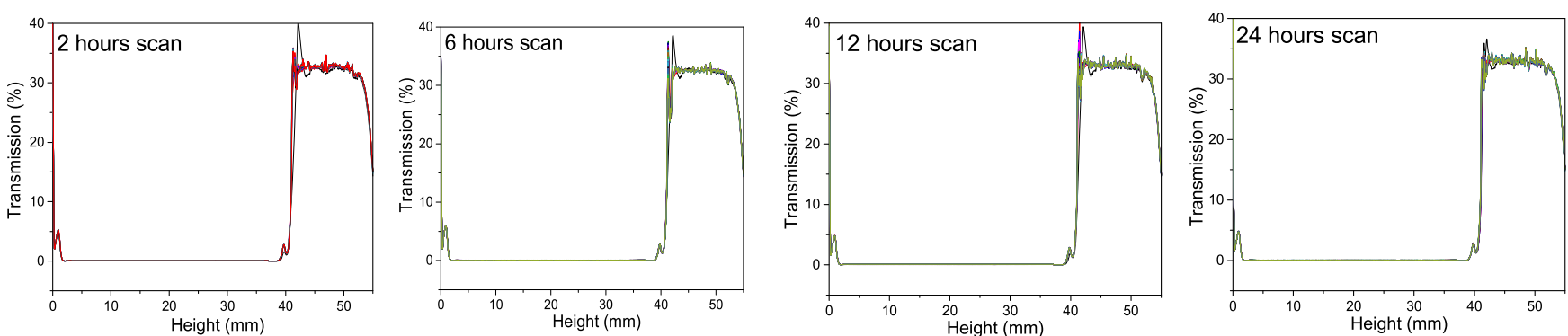

Figure 5. Initial stability test observed using a turbiscan analyzer to understand the extent of sedimentation of GO-COPAM in (a) APIB and (b) FB and zwitterionic rGO-COPAM in (c) APIB and (d) FB after preparation, with scan every 5 min for the duration of, 2, 6, 12, and 24 h.

of the zwitterionic rGO-COPAM proposed that some functionalization of the sample has successfully occurred together with the polymer chain alkylation which results after reacting with 1,3-propane sultone. The concentration of zwitterionic rGO-COPAM in aqueous dispersion was obtained when the known volume of the sample was dried in vacuo.

3.2. Dispersion Stability Analysis. The ability to address the challenges of polymer nanoparticle composite stability at high temperature and high ionic strength into oil reservoir conditions is presented in this work. First, in our preliminary evaluation, we pursue to select polyacrylamide and GO nanosheets as a nanocomposite of interest to produce a modified composite that is stabilized under high ionic strength. To establish the guideline for stability and dispersion, two testing brines were selected: (1) APIB and (2) FB, which are normally discovered predominantly in deep oil reservoirs. The composition and relative weight percentage of salts are shown in Table 1. In comparison to APIB, FB possesses a much complex variety of salts and unquestionably greater number of divalent ions. A preliminary study was initiated on the 

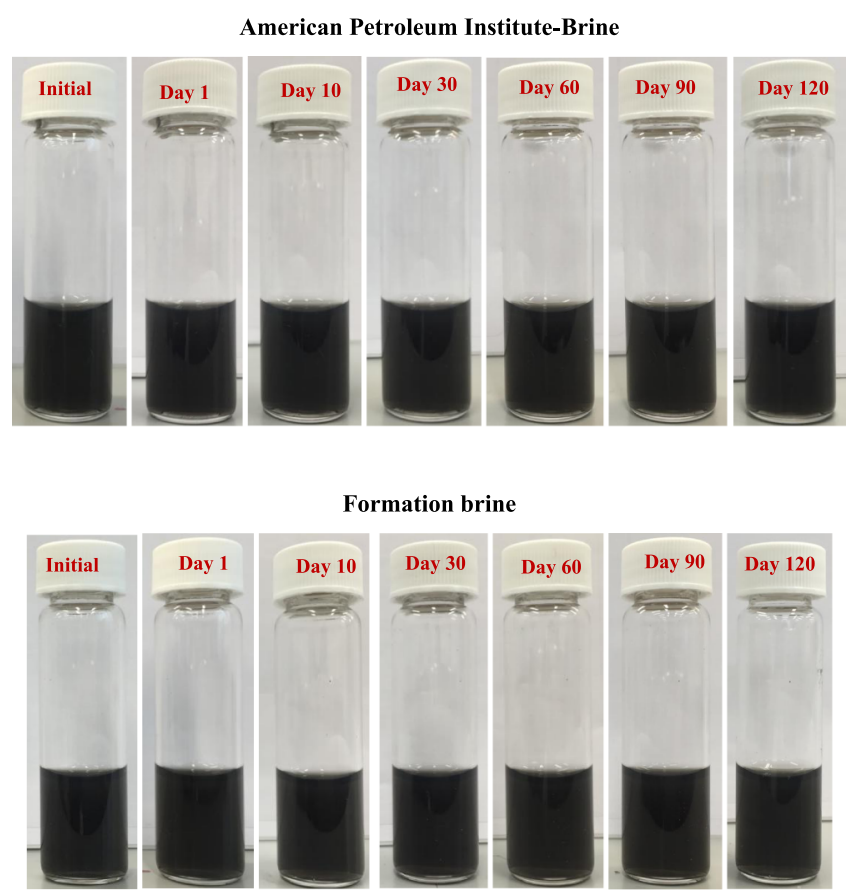

Figure 6. Image of the portion of stable composite dispersions taken after storage at $80{ }^{\circ} \mathrm{C}$ for $0,1,10,30,60,90$, and 120 days in the presence of APIB (top) and FB (bottom).

synthesized composite (GO-COPAM), which reveals that the composite was unstable in both mixtures of APIB and FB after sample preparation stage and aging for 1 day at $80{ }^{\circ} \mathrm{C}$, as illustrated in Figure 4. This is due to the electrostatic crosslinking between GO sheets caused by divalent ions and consequently led to destabilization of the solution.

As discussed in the Introduction section, zwitterionic species and poly-zwitterions provide good dispersibility in harsh conditions such as high ionic strength brines because they exhibit the antipolyelectrolyte effect. ${ }^{29}$ Therefore, a copolymer containing sulfobetaine zwitterionic groups was developed in this project. In the first instance, a COPAM of AA and ATAC were prepared via free-radical polymerization at $80{ }^{\circ} \mathrm{C}$ for $6 \mathrm{~h}$ using ACPA as the initiator, as shown in Scheme 1 (stage 1). The interaction between COPAM and GO sheets occurred through hydrogen bonding and covalent functionalization of amino groups in the polymer chain producing a covalent attachment with graphene basal plane as depicted by FTIR analysis as shown in Figure 1.
In the first place, GO was partially reduced through the interaction of L-ascorbic acid to enhance the GO graphitic domains that improve the stability and enable interaction with the polymer chains. The cooled solution of partial rGO was later added to the COPAM solution as illustrated in Scheme 1 (stage 2). The obtained $\mathrm{rGO}-\mathrm{COPAM}$ was separated by precipitation, cleaned, as well as a further consequent reaction was carried out with 1,3-propane sultone, which lead to the formation of zwitterionic character on the rGO-COPAM composites (zwitterionic rGO-COPAM), as can be seen in Scheme 1 (stage 3 ). It would be expected from the purely zwitterionic polymer to be charge free, but this new zwitterionic $\mathrm{rGO}-$ COPAM probably has anionic groups arising from the sheets of rGO enabling material stabilization. In our finding, the zeta potential (Figure $8 \mathrm{~b}$ ) showed that the surface charge of the prepared material is anionic in nature (negative charge), which is due to the ionization of phenolic and carboxylic acid groups in the solution, ${ }^{5,56,57}$ confirming that the formation of stable GO-COPAM solution should be promoted not just by hydrophilicity of GO but the electrostatic repulsion.

The long-term thermal stability and dispersibility of the aqueous zwitterionic rGO-COPAM mixture in APIB and FB at $80{ }^{\circ} \mathrm{C}$ was investigated. Composites $(10 \mathrm{~mL})$ were added to $90 \mathrm{~mL}$ of each of the individual brine solution to obtain $100 \mathrm{~mL}$ of mixture. Sonication of the mixture was conducted for $5 \mathrm{~min}$ to obtain a homogeneous dispersion, whose aggregates are free after visual inspection. Before aging the samples, both GO-COPAM and zwitterionic rGO-COPAM composites undergo initial stability testing using the turbiscan analyzer to monitor the sedimentation behavior by the transmission or backscattering profile against the sample height with scan every $5 \mathrm{~min}$ for different time intervals $(2,6,12$, and $24 \mathrm{~h}$ ), as shown in Figure 5, while transmission and backscattering data are presented in Figures S5 and S6. The transmission/ backscattering profiles use the light rays to capture the changes in the particle sedimentation within the measuring cell. At a certain interval of time, when sedimentation occurs, the changes in transmission or backscattering vary with the sample height. In this work, the GO-COPAM sample showed significant sedimentation in both APIB and FB after $24 \mathrm{~h}$ scan, as shown in Figure 5a,b, whereas the zwitterionic rGO-COPAM composites displayed excellent stability in both APIB and FB throughout the scanning period, as can be seen in Figure 5c,d.

To understand the long-term stability of zwitterionic rGOCOPAM composites, the solution was stored and left undisturbed at $80{ }^{\circ} \mathrm{C}$ for 120 days. A small portion of each
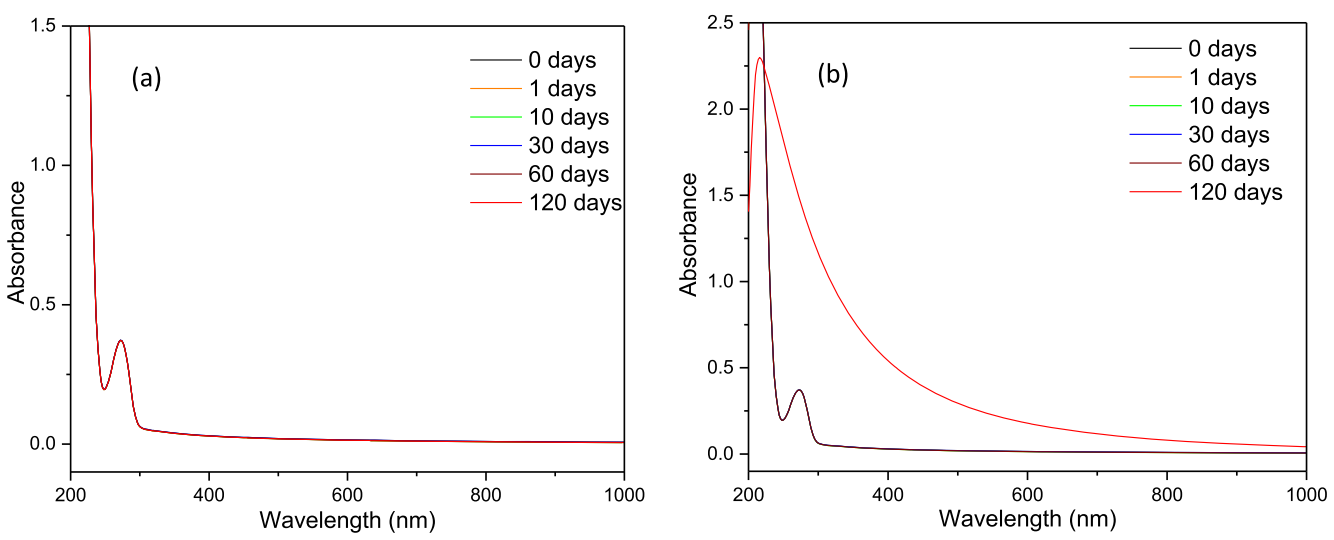

Figure 7. UV-vis spectra of zwitterionic rGO-COPAM solution after 1, 10, 30, 60, 90, and 120 days of aging at $80{ }^{\circ} \mathrm{C}$ in $\mathrm{APIB}$ (a) and FB (b). 

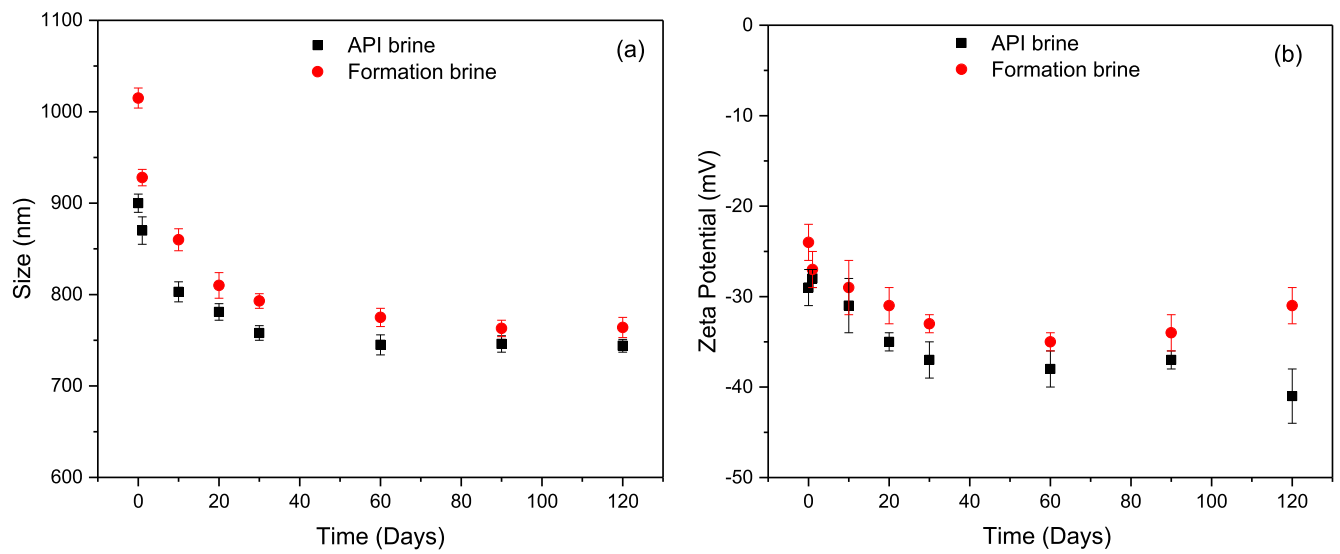

Figure 8. Hydrodynamic size (a) and zeta potential analysis (b) of zwitterionic rGO-COPAM solution after 0, 1, 10, 30, 60, 90, and 120 days of aging at $80^{\circ} \mathrm{C}$ in APIB and FB.
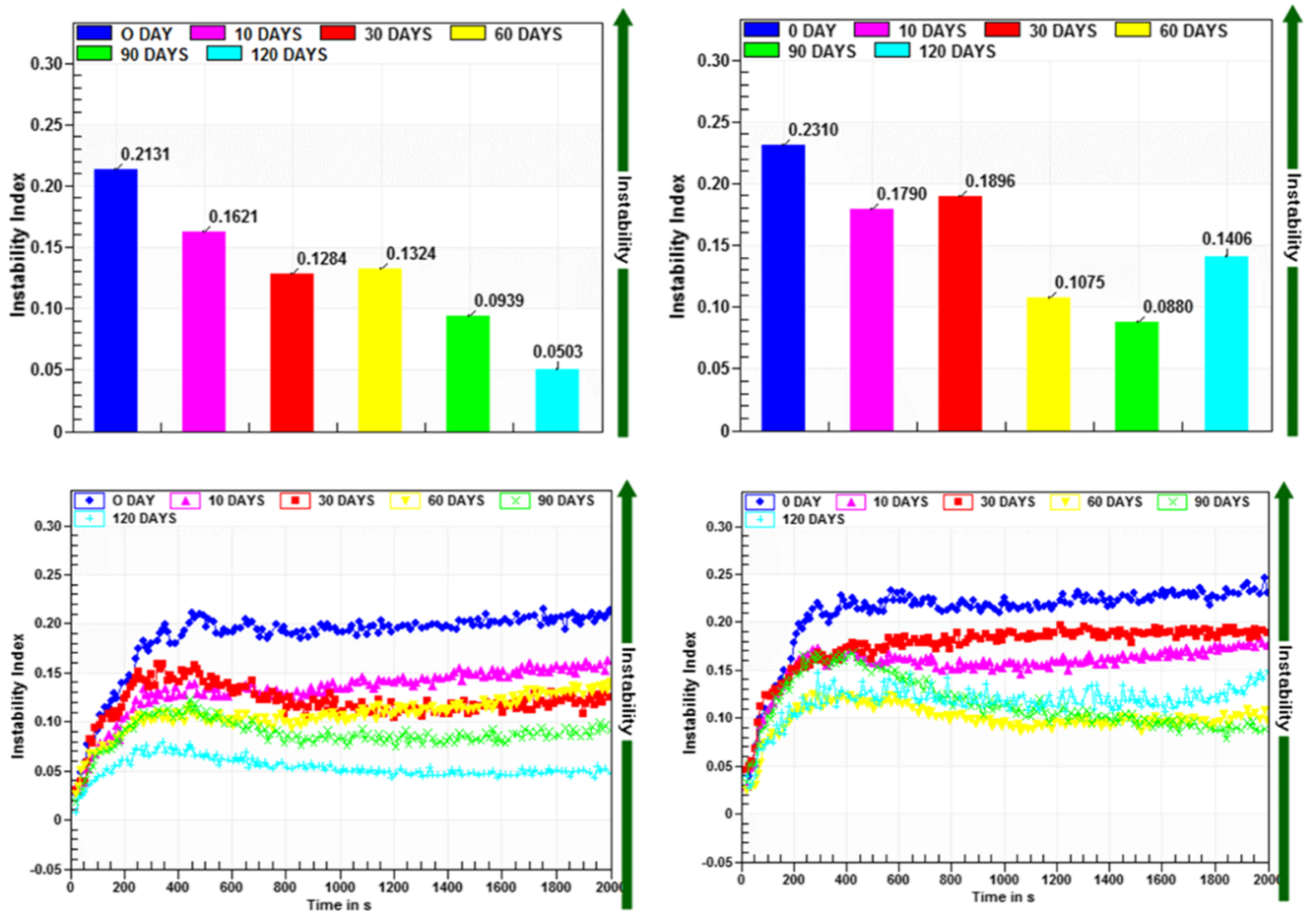

Figure 9. Trends of instability index versus time for zwitterionic rGO-COPAM dispersion in the presence of APIB (left) and FB (right) after 0, $10,30,60,90$, and 120 days of aging at $80^{\circ} \mathrm{C}$.

of the prepared dispersion was taken for spectroscopic analysis at the initial preparation stage and after 1 day $(24 \mathrm{~h})$, followed by $10,30,60,90$, and 120 days for the purpose of characterization. Photos of the aliquots are taken for visual stability test and no notable indication of settling, sedimentation, or instability of the zwitterionic $\mathrm{rGO}-\mathrm{COPAM}$ dispersion was observed in each of the APIB or FB, as shown in Figure 6. However, for quantitative analysis, we first examined the dispersion stability by measuring the UV-vis spectroscopy of the samples. As can be seen in Figure 7, the results at the preparation stage and after aging until 120 days at $80{ }^{\circ} \mathrm{C}$ showed a slight difference in the APIB solution, but changes was observed in FB dispersion especially at 120 days of storage, showing a superimposable character where the peak of GO dispersion shifted from $>230$, and the absorption in the whole spectral region increases with time, proving that the composite stability in the FB reduces at 120 days.
The stability of the zwitterionic rGO-COPAM composite at high temperature in the brines was further confirmed by the changes in hydrodynamic diameter (HD) or size and zeta potential using DLS technique, as shown in Figure 8. The DLS results revealed that the average $\mathrm{HD}$ of the pure $\mathrm{GO}$ sheet in water was $701 \pm 80 \mathrm{~nm}$. At the initial preparation stage of the composite, the average HD was found to be $900 \pm 10$ and $1015 \pm 11 \mathrm{~nm}$ in the API and FB brine solution, respectively. However, the diameters reduce to 870 and 928 after 1 day of aging, displaying a good stability with time, showing a diameter approximately $750 \mathrm{~nm}$ over the storage period in both APIB and FB. Zeta potential analysis also showed the improvement in stability with zwitterionic $\mathrm{rGO}-\mathrm{COPAM}$ composite at both APIB and FB over the storage time, and it was also observed that the zeta potential value of both solutions start to improve after aging at $80^{\circ} \mathrm{C}$ compared to that of the initial preparation, which became approximately $-38 \pm 3 \mathrm{mV}$ in APIB and $-32 \pm 2$ 
in FB. However, in FB after 120 days of aging, the zeta potential decreases to around $-24 \pm 2 \mathrm{mV}$, which is consistent with the obtained UV-vis spectroscopy analysis results. The composite dispersion in brine was assumed to agree with the behavior of pseudo-spherical nanoparticles where the rGO nanosheets wrapped by the chains of zwitterionic COPAM. At high ionic strength brines, the presence of electrolyte ions can enhance the interactions of the ionic groups in the zwitterionic sulfobetaine polymers, creating the chain expansion by providing adequate steric repulsion, thereby preventing the flocculation/aggregation of the composite materials. ${ }^{27,29}$

The dispersion stability of zwitterionic $\mathrm{rGO}-\mathrm{COPAM}$ was also investigated using LUMiSizer-6110 to understand the sedimentation behavior as described in the literature. ${ }^{58,59}$ As we can see from Figures 9 and S7, the stability of the dispersion become more obvious with aging in both APIB and FB, although after 120 days in FB, the instability index starts to increase, which is also supporting the observation made in UVvis and zeta potential analysis. This is probably because the GO nanosheets start to agglomerate after 120 days because of the presence of complex salt, thereby creating higher open particle bed structure, which aggregated more by the applied centrifugal force. Overall, based on the visual and spectroscopic analysis, the zwitterionic rGO-COPAM displayed an outstanding and promising stability in the presence of high-ionic-strength brines including $\mathrm{CaCl}_{2}$ and $\mathrm{MgCl}_{2}$ because there is no observable flocculation or precipitation and only negligible differences were observed over time in the monitored transmittance. Therefore, it could be revealed that the relative stability of the composite dispersion may have likely prolonged above 120 days in $\mathrm{APIB}^{28}$ because up to the storage period spent in this experiment, there was negligible formation in the destabilization of the formed materials, but no longer than 90 days in complex FB.

\section{CONCLUSIONS}

Stabilization of polymer nanocomposites in high-ionic-strength brines is a challenging issue. This study developed a new way to enhance the stability of GO polyacrylamide nanocomposites at high-temperature and high-ionic-strength brines. COPAM was covalently attached to the partial $\mathrm{rGO}$ via free-radical polymerization and reacted further with 1,3-propane sultone to accomplish zwitterionic groups. Compared to GO-COPAM, zwitterionic rGO-COPAM dispersions showed that no noticeable sedimentation or flocculation observed, the particle size measured was smaller with large zeta potentials, and the instability index derived from LUMiSizer was smaller over a storage of 120 days at $80{ }^{\circ} \mathrm{C}$ under both APIB and FB conditions. It also showed that the dispersion stability was better in APIB than in FB, where the GO-sheet conjugated structure may have been altered after 90 days in the FB. FTIR and other chemical analysis suggested that the formation of zwitterionic character on the $\mathrm{GGO}-\mathrm{COPAM}$ composites was responsible for the excellent temperature stability and dispersibility in the presence of high salinity and high temperature. Such results suggest that the zwitterionic molecules could be used as a stability enhancer for different particles, and the zwitterionic polyacrylamide-GO systems possess greater potential for future enhanced oil recovery applications.

\section{ASSOCIATED CONTENT}

\section{S Supporting Information}

The Supporting Information is available free of charge on the ACS Publications website at DOI: 10.1021/acsomega.9b00963.
Experimental setup for polymerization process; solubility and viscosity of pure COPAM with different concentrations of 1,3 propane sultone; NMR analysis of COPAM synthesized via free-radical polymerization; XPS spectrum of GO-COPAM showing the presence of nitrogen, oxygen, and carbon and zwitterionic rGOCOPAM composites illustrating the presence of sulfur, nitrogen, oxygen, and carbon; presence of $\mathrm{Mn}$ peak in GO-COPAM spectrum is presumed to emerge from the trace of $\mathrm{KMnO}_{4}$ in $\mathrm{GO}$ synthesis or possible contamination from glassware; initial stability test observed using the turbiscan analyzer to understand the extent of sedimentation of GO-COPAM and zwitterionic rGO-COPAM in APIB after preparation, with scan every $5 \mathrm{~min}$ for the duration of, 2, 6, 12, and $24 \mathrm{~h}$, respectively, showing both transmission and backscattering profiles; and styles of light transmission across the test tube for zwitterionic rGO-COPAM dispersion in the presence of APIB and FB after 0, 10, $30,60,90$, and 120 days of aging at $80{ }^{\circ} \mathrm{C}$ (PDF)

\section{AUTHOR INFORMATION}

\section{Corresponding Author}

*E-mail: d.wen@leeds.ac.uk and d.wen@buaa.edu.cn. Phone: +44(0)1133431299.

ORCID $\odot$

Maje Alhaji Haruna: 0000-0003-3504-1876

Dongsheng Wen: 0000-0003-3492-7982

Notes

The authors declare no competing financial interest.

\section{ACKNOWLEDGMENTS}

The authors would like to thank the support of Petroleum Technology Development Funds (PTDF) in Nigeria for merit $\mathrm{PhD}$ scholarship and the European Research Council (ERC2014-CoG, Project reference: 648375). The authors would like to extend their sincere thanks to Dr. Jabbar Gardy, Shahid Pervaiz and Dr. Antonios Anastasiou for their keen support toward the completion of this work.

\section{REFERENCES}

(1) Hwang, C.-C.; et al. Highly stable carbon nanoparticles designed for downhole hydrocarbon detection. Energy Environ. Sci. 2012, 5, 8304-8309.

(2) Vasantha, V. A.; et al. Water swelling, brine soluble imidazole based zwitterionic polymers - synthesis and study of reversible UCST behaviour and gel-sol transitions. Chem. Commun. 2014, 50, 46-48.

(3) Bagaria, H. G.; Johnston, K. P.; et al. Iron oxide nanoparticles grafted with sulfonated copolymers are stable in concentrated brine at elevated temperatures and weakly adsorb on silica. ACS Appl. Mater. Interfaces 2013, 5, 3329-3339.

(4) Geim, A. K.; Novoselov, K. S. The rise of graphene. Nat. Mater. 2007, 6, 183.

(5) Li, D.; et al. Processable aqueous dispersions of graphene nanosheets. Nat. Nanotechnol. 2008, 3, 101.

(6) Novoselov, K. S.; et al. Electric field effect in atomically thin carbon films. science 2004, 306, 666-669.

(7) Cheraghian, G. Synthesis and properties of polyacrylamide by nanoparticles, effect nanoclay on stability polyacrylamide solution. Micro Nano Lett. 2017, 12, 40-44.

(8) Cheraghian, G. Thermal resistance and application of nanoclay on polymer flooding in heavy oil recovery. Pet. Sci. Technol. 2015, 33, $1580-1586$ 
(9) Cheraghian, G. Evaluation of clay and fumed silica nanoparticles on adsorption of surfactant polymer during enhanced oil recovery. J. Jpn. Pet. Inst. 2017, 60, 85-94.

(10) Cheraghian, G. Effects of titanium dioxide nanoparticles on the efficiency of surfactant flooding of heavy oil in a glass micromodel. Pet. Sci. Technol. 2016, 34, 260-267.

(11) Cheraghian, G.; Tardasti, S. Improved oil recovery by the efficiency of nano-particle in imbibition mechanism. 2nd EAGE International Conference KazGeo, 2012.

(12) Liu, R.; et al. Tough and highly stretchable graphene oxide/ polyacrylamide nanocomposite hydrogels. J. Mater. Chem. 2012, 22, 14160-14167.

(13) Liu, J.; et al. Self-Healing in Tough Graphene Oxide Composite Hydrogels. Macromol. Rapid Commun. 2013, 34, 1002-1007.

(14) Shen, J.; et al. Study on graphene-oxide-based polyacrylamide composite hydrogels. Composites, Part A 2012, 43, 1476-1481.

(15) Adhikari, B.; Biswas, A.; Banerjee, A. Graphene oxide-based supramolecular hydrogels for making nanohybrid systems with $\mathrm{Au}$ nanoparticles. Langmuir 2011, 28, 1460-1469.

(16) Bai, H.; et al. Graphene oxide/conducting polymer composite hydrogels. J. Mater. Chem. 2011, 21, 18653-18658.

(17) Kuilla, T.; et al. Recent advances in graphene based polymer composites. Prog. Polym. Sci. 2010, 35, 1350-1375.

(18) Potts, J. R.; et al. Graphene-based polymer nanocomposites. Polymer 2011, 52, 5-25.

(19) Fan, J.; et al. Glycidyl methacrylate-modified gum arabic mediated graphene exfoliation and its use for enhancing mechanical performance of hydrogel. Polymer 2013, 54, 3921-3930.

(20) Yang, Y.; et al. Preparation of Reduced Graphene Oxide/ Poly(acrylamide) Nanocomposite and Its Adsorption of $\mathrm{Pb}(\mathrm{II})$ and Methylene Blue. Langmuir 2013, 29, 10727-10736.

(21) Nguyen, B. D.; et al. The impact of graphene oxide particles on viscosity stabilization for diluted polymer solutions using in enhanced oil recovery at HTHP offshore reservoirs. Adv. Nat. Sci.: Nanosci. Nanotechnol. 2014, 6, 015012.

(22) Haruna, M. A.; et al. Improved rheology and high-temperature stability of hydrolyzed polyacrylamide using graphene oxide nanosheet. J. Appl. Polym. Sci. 2019, 136, 47582.

(23) McCoy, T. M.; Pottage, M. J.; Tabor, R. F. Graphene oxidestabilized oil-in-water emulsions: $\mathrm{pH}$-controlled dispersion and flocculation. J. Phys. Chem. C 2014, 118, 4529-4535.

(24) Yoon, K. Y.; et al. Graphene oxide nanoplatelet dispersions in concentrated $\mathrm{NaCl}$ and stabilization of oil/water emulsions. J. Colloid Interface Sci. 2013, 403, 1-6.

(25) Stankovich, S.; et al. Synthesis of graphene-based nanosheets via chemical reduction of exfoliated graphite oxide. carbon 2007, 45, $1558-1565$

(26) McAllister, M. J.; et al. Single sheet functionalized graphene by oxidation and thermal expansion of graphite. Chem. Mater. 2007, 19, 4396-4404.

(27) Lowe, A. B.; McCormick, C. L. Synthesis and Solution Properties of Zwitterionic Polymers $\dagger$. Chem. Rev. 2002, 102, 41774190.

(28) Zuniga, C. A.; et al. Long-Term High-Temperature Stability of Functionalized Graphene Oxide Nanoplatelets in Arab-D and API Brine. ACS Appl. Mater. Interfaces 2016, 8, 1780-1785.

(29) Israelachvili, J. N. Intermolecular and Surface Forces; Academic Press, 2011.

(30) Quintana, R.; et al. Sulfobetaine-based polymer brushes in marine environment: Is there an effect of the polymerizable group on the antifouling performance? Colloids Surf., B 2014, 120, 118-124.

(31) Wever, D. A. Z.; Picchioni, F.; Broekhuis, A. A. Polymers for enhanced oil recovery: A paradigm for structure-property relationship in aqueous solution. Prog. Polym. Sci. 2011, 36, 1558-1628.

(32) Compton, O. C.; Nguyen, S. T. Graphene Oxide, Highly Reduced Graphene Oxide, and Graphene: Versatile Building Blocks for Carbon-Based Materials. small 2010, 6, 711-723.

(33) Dreyer, D. R.; Todd, A. D.; Bielawski, C. W. Harnessing the chemistry of graphene oxide. Chem. Soc. Rev. 2014, 43, 5288-5301.
(34) Wang, H.; et al. Graphene-Wrapped Sulfur Particles as a Rechargeable Lithium-Sulfur Battery Cathode Material with High Capacity and Cycling Stability. Nano Lett. 2011, 11, 2644-2647.

(35) Sreejith, S.; Ma, X.; Zhao, Y. Graphene oxide wrapping on squaraine-loaded mesoporous silica nanoparticles for bioimaging. $J$. Am. Chem. Soc. 2012, 134, 17346-17349.

(36) Ma, X.; et al. Graphene oxide wrapped gold nanoparticles for intracellular Raman imaging and drug delivery. J. Mater. Chem. B 2013, 1, 6495-6500.

(37) Nguyen, K. T.; Zhao, Y. Integrated graphene/nanoparticle hybrids for biological and electronic applications. Nanoscale 2014, 6, $6245-6266$.

(38) Hummers, W. S., Jr.; Offeman, R. E. Preparation of graphitic oxide. J. Am. Chem. Soc. 1958, 80, 1339.

(39) Haruna, M. A.; et al. Improved Polymer Flooding in Harsh Environment by Free-Radical Polymerization and the Use of Nanomaterials. Energy Fuels 2019, 33, 1637.

(40) Nourafkan, E.; et al. Improved rheological properties and stability of multiwalled carbon nanotubes/polymer in harsh environment. J. Appl. Polym. Sci. 2018, 136, 47205.

(41) Hebishy, E.; et al. Physical and oxidative stability of whey protein oil-in-water emulsions produced by conventional and ultra high-pressure homogenization: Effects of pressure and protein concentration on emulsion characteristics. Innovative Food Sci. Emerging Technol. 2015, 32, 79-90.

(42) Mahdavian, A.-R.; Abdollahi, M.; Bijanzadeh, H. R. Kinetic study of radical polymerization. III. Solution polymerization of acrylamide by1H-NMR. J. Appl. Polym. Sci. 2004, 93, 2007-2013.

(43) Cheng, Q.-Y.; et al. Supramolecular self-assembly induced graphene oxide based hydrogels and organogels. Langmuir 2012, 28, 3005-3010.

(44) Wang, X.; Hu, D.; Yang, J. Synthesis of PAM/TiO2 composite microspheres with hierarchical surface morphologies. Chem. Mater. 2007, 19, 2610-2621.

(45) Liao, Y.; et al. UV-initiated polymerization of hydrophobically associating cationic polyacrylamide modified by a surface-active monomer: a comparative study of synthesis, characterization, and sludge dewatering performance. Ind. Eng. Chem. Res. 2014, 53, 11193-11203.

(46) Li, X.; et al. Optimized preparation of micro-block CPAM by response surface methodology and evaluation of dewatering performance. RSC Adv. 2017, 7, 208-217.

(47) Ni, Y.; et al. Preparation, characterization, and optical, electrochemical property research of CdS/PAM nanocomposites. J. Phys. Chem. B 2006, 110, 17347-17352.

(48) Hu, Z.; et al. Rheological Properties of Partially Hydrolyzed Polyacrylamide Seeded by Nanoparticles. Ind. Eng. Chem. Res. 2017, 56, 3456-3463.

(49) Gao, H.; et al. Bifunctional ultraviolet/ultrasound responsive composite $\mathrm{TiO} 2 /$ polyelectrolyte microcapsules. Nanoscale 2016, 8, $5170-5180$

(50) Durmaz, S.; Okay, O. Acrylamide/2-acrylamido-2-methylpropane sulfonic acid sodium salt-based hydrogels: synthesis and characterization. Polymer 2000, 41, 3693-3704.

(51) Rosa, F.; Bordado, J.; Casquilho, M. Hydrosoluble copolymers of acrylamide-(2-acrylamido-2-methylpropanesulfonic acid). Synthesis and characterization by spectroscopy and viscometry. J. Appl. Polym. Sci. 2003, 87, 192-198.

(52) Xu, L.; et al. Synthesis and thermal degradation property study of N-vinylpyrrolidone and acrylamide copolymer. RSC Adv. 2014, 4, 33269-33278.

(53) Haruna, M. A.; et al. Influence of carbon quantum dots on the viscosity reduction of polyacrylamide solution. Fuel 2019, 248, 205214.

(54) Avşar, A.; et al. A novel catalyst system for the synthesis of $\mathrm{N}$, $\mathrm{N}^{\prime}$-Methylenebisacrylamide from acrylamide. Des. Monomers Polym. 2017, 20, 434-440. 
(55) Cao, J.; et al. Application of Amino-Functionalized Nanosilica in Improving the Thermal Stability of Acrylamide-Based Polymer for Enhanced Oil Recovery. Energy Fuels 2018, 32, 246-254.

(56) Lerf, A.; et al. Structure of Graphite Oxide Revisited\|. J. Phys. Chem. B 1998, 102, 4477-4482.

(57) Szabó, T.; et al. Evolution of surface functional groups in a series of progressively oxidized graphite oxides. Chem. Mater. 2006, $18,2740-2749$.

(58) Walter, J.; et al. New possibilities of accurate particle characterisation by applying direct boundary models to analytical centrifugation. Nanoscale 2015, 7, 6574-6587.

(59) Chiu, H.-T.; et al. Using analytical centrifugation to characterize the dispersibility and particle size distributions of organic/inorganic composite coatings. J. Polym. Res. 2011, 18, $1587-1596$ 